\title{
On Extremal Ranks and Least Squares Solutions Subject to a Rank Restriction
}

\author{
Hongxing Wang and Yeguo Sun \\ Department of Mathematics and Computational Science, Huainan Normal University, Anhui 232038, China \\ Correspondence should be addressed to Yeguo Sun; ygsun@hnnu.edu.cn
}

Received 16 February 2014; Revised 11 June 2014; Accepted 22 June 2014; Published 8 July 2014

Academic Editor: Sofiya Ostrovska

Copyright (C) $2014 \mathrm{H}$. Wang and Y. Sun. This is an open access article distributed under the Creative Commons Attribution License, which permits unrestricted use, distribution, and reproduction in any medium, provided the original work is properly cited.

We discuss the feasible interval of the parameter $k$ and a general expression of matrix $X$ which satisfies the rank equation $r(A-$ $B X C)=k$. With these results, we study two problems under the rank constraint $r(A-B X C)=k$. The first one is to determine the maximal and minimal ranks under the rank constraint $r(A-B X C)=k$. The second one is to derive the least squares solutions of $\|B X C-A\|_{F}=$ min under the rank constraint $r(A-B X C)=k$.

\section{Introduction}

We adopt the following notation in this paper. The set of $m \times n$ matrices with complex entries is denoted by $\mathbb{C}^{m \times n}$. The conjugate transpose of a matrix $A$ is denoted by $A^{*}$. The symbols $I_{k}$ and $r(A)$ are the $k \times k$ identity matrix and the rank of $A \in \mathbb{C}^{m \times n}$, respectively. $\|\cdot\|$ stands for the matrix Frobenius norm. The Moore-Penrose inverse of $A \in \mathbb{C}^{m \times n}$ is defined as the unique matrix $X \in \mathbb{C}^{n \times m}$ satisfying

$$
\begin{array}{ll}
\text { (1) } A X A=A, & \text { (2) } X A X=X, \\
\text { (3) }(A X)^{*}=A X, & \text { (4) }(X A)^{*}=X A,
\end{array}
$$

and is denoted by $X=A^{\dagger}$ (see [1]). Furthermore, we denote $E_{A}=I_{m}-A A^{\dagger}$ and $F_{A}=I_{n}-A^{\dagger} A$.

In the literature, ranks of solutions of linear matrix equations have been studied widely. Uhlig [2] derived the extremal ranks of solutions of the consistent matrix equation of $A X=B$. Tian [3] derived the extremal ranks of solutions of $B X C=A . \mathrm{Li}$ and Liu [4] studied the extremal ranks of Hermitian solutions of $A X=B$. Li et al. [5] studied the extremal ranks of solutions with special structure of $A X=$ $B$. Liu [6] derived the extremal ranks of solutions of $A X+$ $Y B=C$. Wang and Li [7] established the maximal and minimal ranks of the solution to consistent system $A_{1} X_{1}=$ $C_{1}, A_{2} X_{2}=C_{2}$, and $A_{3} X_{1} B_{1}+A_{4} X_{2} B_{2}=C_{3}$. Wang and
He [8] derived the extremal ranks of the general solution of the mixed Sylvester matrix equations

$$
\begin{aligned}
& A_{1} X-Y B_{1}=C_{1}, \\
& A_{2} Z-Y B_{2}=C_{2} .
\end{aligned}
$$

Liu [9] derived the extremal ranks of least square solutions to $B X C=A$. Sou and Rantzer [10] studied the minimum rank matrix approximation problem in the spectral norm

$$
\min _{X} \operatorname{rank}(X) \quad \text { subject to }\|A-B X C\|_{2}<1 .
$$

Wei and Shen [11] studied a more general problem

$$
\min _{X} \operatorname{rank}(X) \quad \text { subject to }\|A-B X C\|_{2}<\xi,
$$

where $\xi \geq \theta$ and $\theta=\min _{Y}\|A-B Y C\|_{2}$. More results and applications about ranks of matrix expressions and solutions of matrix equations can be seen in ([2, 3, 8, 11-13], etc.).

Motivated by the work of $[2,3,7-9,14,15]$, we consider a general problem. Assume that $k$ is a prescribed nonnegative integer and $A \in \mathbb{C}^{m \times n}, B \in \mathbb{C}^{m \times p}$, and $C \in \mathbb{C}^{q \times n}$ are given matrices. We now investigate the problem to determine the maximal and minimal ranks of solutions to the rank equation $r(A-B X C)=k$. This problem can be stated as follows.

Problem 1. Given matrices $A \in \mathbb{C}^{m \times n}, B \in \mathbb{C}^{m \times p}$, and $C \in$ $\mathbb{C}^{q \times n}$ and nonnegative integer $k_{1}$, characterize the set

$$
\mathbb{S}_{k_{1}}=\left\{X \mid X \in \mathbb{C}^{p \times q}, r(A-B X C)=k_{1}\right\},
$$


and determine the maximal and minimal ranks of solutions of the rank equation $r(A-B X C)=k_{1}$.

In [16-18], Wang, Wei, and Zha studied least squares solutions of line matrix equations under rank constraints, respectively. In [19], Wei and Wang derived a rank- $k$ Hermitian nonnegative definite least squares solution to the equation $B X B^{*}=A$. In Problem 2, we discuss the least squares solutions $X$ of $\|B X C-A\|_{F}=$ min subject to $r(A-$ $B X C)=k$. This problem can be stated as follows.

Problem 2. Given matrices $A \in \mathbb{C}^{m \times n}, B \in \mathbb{C}^{m \times p}$, and $C \in \mathbb{C}^{q \times n}$ and nonnegative integer $k$, determine the range of $k$, such that there exists a least squares solution $X$ of $\|B X C-A\|_{F}=$ min subject to $r(A-B X C)=k$; that is, characterize the set

$$
\begin{aligned}
\widehat{\mathbb{S}}=\left\{X \mid X \in \mathbb{C}^{p \times q},\right. \\
\|A-B X C\|=\text { min subject to } r(A-B X C)=k\} .
\end{aligned}
$$

The paper is organized as follows. In Section 2, we provide some preliminary results; in Sections 3 and 4, we study Problems 1 and 2, respectively; and finally in Section 5, we conclude the paper with some remarks.

\section{Preliminaries}

In this section we present some preliminary results which will be used in the following sections to study Problems 1 and 2.

Lemma 3 (see [20]). Let $A \in \mathbb{C}^{m \times n}, B \in \mathbb{C}^{m \times k}, C \in \mathbb{C}^{l \times n}$, and $D \in \mathbb{C}^{l \times k}$ be given. Then

$$
\begin{gathered}
r\left[\begin{array}{ll}
A & B
\end{array}\right]=r(A)+r\left(E_{A} B\right)=r(B)+r\left(E_{B} A\right), \\
r\left[\begin{array}{l}
A \\
C
\end{array}\right]=r(A)+r\left(C F_{A}\right)=r(C)+r\left(A F_{C}\right), \\
\quad r\left[\begin{array}{ll}
A & B \\
C & 0
\end{array}\right]=r(B)+r(C)+r\left(E_{B} A F_{C}\right),
\end{gathered}
$$

where $B_{1}=E_{A} B$ and $C_{1}=C F_{A}$.

Lemma 4. Let $A \in \mathbb{C}^{m \times n}$ be given. Then

$$
\begin{gathered}
\min _{r(X)=k} r(A-X)=\max \{k-r(A), r(A)-k\}, \\
\max _{r(X)=k} r(A-X)=\min \{m, n, k+r(A)\} .
\end{gathered}
$$

Lemma 5 (see [21]). Let $A \in \mathbb{C}^{m \times n}, B \in \mathbb{C}^{m \times p}, C \in \mathbb{C}^{q \times n}$, and $D \in \mathbb{C}^{q \times p}$ be given. Then

$$
r\left(D-C A^{\dagger} B\right)=r\left[\begin{array}{cc}
A^{*} A A^{*} & A^{*} B \\
C A^{*} & D
\end{array}\right]-r(A)
$$

Lemma 6 (see [22, 23] (the Eckart-Young-Mirsky theorem)). Let $A \in \mathbb{C}_{r}^{m \times n}, k$ be a given nonnegative integer in which $k \leq r$ and the singular value decomposition [24] of $A$ be

$$
A=U\left[\begin{array}{ll}
\Sigma & 0 \\
0 & 0
\end{array}\right] V^{*},
$$

where $\Sigma=\operatorname{diag}\left\{\lambda_{1}, \ldots, \lambda_{r}\right\}, \lambda_{1} \geq \cdots \geq \lambda_{r}>0$, and $U$ and $V$ are unitary matrices of appropriate sizes. Then

$$
\min _{r(X)=k}\|A-X\|^{2}=\sum_{i=k+1}^{r} \lambda_{i}^{2} .
$$

Furthermore, when $\lambda_{k}>\lambda_{k+1}$,

$$
X=U \operatorname{diag}\left\{\lambda_{1}, \ldots, \lambda_{k}, 0, \ldots, 0\right\} V^{*} ;
$$

when $p<k<q \leq r$ and $\lambda_{p}>\lambda_{p+1}=\cdots=\lambda_{q}>\lambda_{q+1}$,

$$
X=U \operatorname{diag}\left\{\lambda_{1}, \ldots, \lambda_{p}, \lambda_{k} Q Q^{*}, 0, \ldots, 0\right\} V^{*} \text {, }
$$

where $Q$ is an arbitrary matrix satisfying $Q \in \mathbb{C}^{(q-p) \times(k-p)}$ and $Q^{*} Q=I_{k-p}$.

\section{Solutions to Problem 1}

In this section, we study Problem 1 proposed in Section 1.

Suppose that the matrices $A \in \mathbb{C}^{m \times n}, B \in \mathbb{C}^{m \times p}$, and $C \in$ $\mathbb{C}^{q \times n}$ are given. Let

$$
l=r\left[\begin{array}{l}
A \\
C
\end{array}\right]+r\left[\begin{array}{ll}
A & B
\end{array}\right]-r\left[\begin{array}{ll}
A & B \\
C & 0
\end{array}\right] .
$$

Then from [25] there exists $X$ such that $r(A-B X C)=k_{1}$, if and only if

$$
\min \left\{r\left[\begin{array}{ll}
A & B
\end{array}\right], r\left[\begin{array}{l}
A \\
C
\end{array}\right]\right\} \geq k_{1} \geq l
$$

Furthermore, let

$$
B=U_{1}\left[\begin{array}{cc}
\Sigma_{1} & 0 \\
0 & 0
\end{array}\right] V_{1}^{*}, \quad C=U_{2}\left[\begin{array}{cc}
\Sigma_{2} & 0 \\
0 & 0
\end{array}\right] V_{2}^{*}
$$

be singular value decompositions of $B$ and $C$ with unitary matrices $U_{1} \in \mathbb{C}^{m \times m}, U_{2} \in \mathbb{C}^{q \times q}, V_{1} \in \mathbb{C}^{p \times p}$, and $V_{2} \in \mathbb{C}^{n \times n}$. Write $U_{1}^{*} A V_{2}$ in partitioned form as

$$
U_{1}^{*} A V_{2}=\left[\begin{array}{ll}
A_{11} & A_{12} \\
A_{21} & A_{22}
\end{array}\right],
$$

where $A_{11} \in \mathbb{C}^{r(B) \times r(C)}, A_{12} \in \mathbb{C}^{r(B) \times(n-r(C))}, A_{21} \in$ $\mathbb{C}^{(m-r(B)) \times r(C)}$, and $A_{22} \in \mathbb{C}^{(m-r(B)) \times(n-r(C))}$. Also assume that the singular value decomposition of $A_{22}$ and the corresponding decompositions are given by

$$
\begin{gathered}
A_{22}=U_{3}\left[\begin{array}{cc}
\Sigma_{3} & 0 \\
0 & 0
\end{array}\right] V_{3}^{*}, \quad U_{3}^{*} A_{21}=\left[\begin{array}{l}
J \\
K
\end{array}\right], \\
A_{12} V_{3}=\left[\begin{array}{ll}
E & F
\end{array}\right], \quad F=U_{4}\left[\begin{array}{cc}
\Sigma_{4} & 0 \\
0 & 0
\end{array}\right] V_{4}^{*}, \\
K=U_{5}\left[\begin{array}{cc}
\Sigma_{5} & 0 \\
0 & 0
\end{array}\right] V_{5}^{*}, \\
U_{4}^{*}\left(A_{11}-E \Sigma_{3}^{-1} J\right) V_{5}=\left[\begin{array}{ll}
\widehat{A}_{11} & \widehat{A}_{12} \\
\widehat{A}_{21} & \widehat{A}_{22}
\end{array}\right],
\end{gathered}
$$


where $U_{i}$ and $V_{i}$ are unitary matrices of appropriate sizes in which $i=3,4,5, J \in \mathbb{C}^{r\left(A_{22}\right) \times r(C)}, K \in \mathbb{C}^{\left(m-r(B)-r\left(A_{22}\right)\right) \times r(C)}$, $E \in \mathbb{C}^{r(B) \times r\left(A_{22}\right)}, F \in \mathbb{C}^{r(B) \times\left(n-r(C)-r\left(A_{22}\right)\right)}, \widehat{A}_{11} \in \mathbb{C}^{r(F) \times r(K)}$, $\widehat{A}_{12} \in \mathbb{C}^{r(F) \times(r(C)-r(K))}, \widehat{A}_{21} \in \mathbb{C}^{(r(B)-r(F)) \times r(K)}$, and $\widehat{A}_{22} \epsilon$ $\mathbb{C}^{(r(B)-r(F)) \times(r(C)-r(K))}$.

We have the following result.

Theorem 7. Suppose that the singular value decompositions of matrices $B, C, A_{22}, F$, and $K$ are given in (19)-(21). $U_{1}^{*} A V_{2}$, $U_{3}^{*} A_{21}, U_{3}^{*} A_{21}, A_{12} V_{3}$, and $U_{4}^{*}\left(A_{11}-E \Sigma_{3}^{-1} J\right) V_{5}$ have the forms in (20) and (21). If $k$ satisfies (18), then any solution $X$ to the rank equation $r(A-B X C)=k_{1}$ has the form

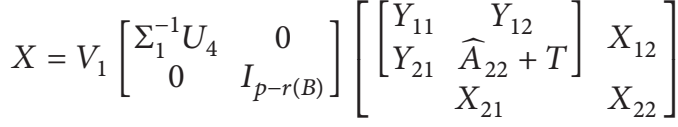

$$
\begin{aligned}
& \times\left[\begin{array}{cc}
V_{5}^{*} \Sigma_{2}^{-1} & 0 \\
0 & I_{q-r(C)}
\end{array}\right] U_{2}^{*},
\end{aligned}
$$

where $X_{12} \in \mathbb{C}^{r(B) \times(q-r(C))}, X_{21} \in \mathbb{C}^{(p-r(B)) \times r(C)}, X_{22} \in$ $\mathbb{C}^{(p-r(B)) \times(q-r(C))}, Y_{11} \in \mathbb{C}^{r(F) \times r(K)}, Y_{12} \in \mathbb{C}^{r(F) \times(r(C)-r(K))}$, and $Y_{21} \in \mathbb{C}^{(r(B)-r(F)) \times r(K)}$ are arbitrary, $T \in \mathbb{C}^{(r(B)-r(F)) \times(r(C)-r(K))}$, and $r(T)=k_{1}-l$.

Proof. From the singular value decompositions of matrices of $B, C, F$, and $K$, we observe that

$$
\begin{aligned}
& U_{1}\left[\begin{array}{cc}
A_{11} & 0 \\
0 & 0
\end{array}\right] V_{2}^{*}=B B^{\dagger} A C^{\dagger} C \\
& U_{1}\left[\begin{array}{cc}
0 & A_{12} \\
0 & 0
\end{array}\right] V_{2}^{*}=B B^{\dagger} A F_{C}, \\
& U_{1}\left[\begin{array}{cc}
0 & 0 \\
A_{21} & 0
\end{array}\right] V_{2}^{*}=E_{B} A C^{\dagger} C, \\
& U_{1}\left[\begin{array}{cc}
0 & 0 \\
0 & A_{22}
\end{array}\right] V_{2}^{*}=E_{B} A F_{C} \\
& U_{1}\left[\begin{array}{ll}
0 & A_{12} \\
0 & A_{22}
\end{array}\right] V_{2}^{*}=A F_{C}, \\
& U_{1}\left[\begin{array}{cc}
0 & 0 \\
A_{21} & A_{22}
\end{array}\right] V_{2}^{*}=E_{B} A \\
& {\left[\begin{array}{ll}
0 & F
\end{array}\right] V_{3}^{*}=A_{12} F_{A_{22}}, \quad U_{3}\left[\begin{array}{c}
0 \\
K
\end{array}\right]=E_{A_{22}} A_{21} \text {, }} \\
& U_{4}\left[\begin{array}{cc}
0 & 0 \\
0 & \widehat{A}_{22}
\end{array}\right] V_{5}^{*}=E_{F}\left(A_{11}-E \Sigma_{3}^{-1} J\right) F_{K} .
\end{aligned}
$$

Then by repeated application of Lemma 3, we have

$$
\begin{gathered}
r\left(A_{22}\right)=r\left(E_{B} A F_{C}\right)=r\left[\begin{array}{ll}
A & B \\
C & 0
\end{array}\right]-r(B)-r(C), \\
r(F)=r\left(\left[\begin{array}{ll}
0 & F
\end{array}\right] V_{3}^{*}\right)=r\left(A_{12} F_{A_{22}}\right)=r\left[\begin{array}{l}
A_{12} \\
A_{22}
\end{array}\right]-r\left(A_{22}\right) \\
=r\left(A F_{C}\right)-r\left(E_{B} A F_{C}\right) \\
=r(B)+r\left[\begin{array}{l}
A \\
C
\end{array}\right]-r\left[\begin{array}{ll}
A & B \\
C & 0
\end{array}\right] \\
r(K)=r\left(U_{3}\left[\begin{array}{l}
0 \\
K
\end{array}\right]\right)=r\left(E_{A_{22}} A_{21}\right) \\
=r\left[\begin{array}{ll}
A_{21} & A_{22}
\end{array}\right]-r\left(A_{22}\right) \\
=r\left(E_{B} A\right)-r\left(E_{B} A F_{C}\right) \\
=r(C)+r[A \quad B]-r\left[\begin{array}{ll}
A & B \\
C & 0
\end{array}\right]
\end{gathered}
$$

Furthermore, write

$$
V_{1}^{*} X U_{2}=\left[\begin{array}{cc}
\Sigma_{1}^{-1} X_{11} \Sigma_{2}^{-1} & X_{12} \\
X_{21} & X_{22}
\end{array}\right], \quad U_{4}^{*} X_{11} V_{5}=\left[\begin{array}{cc}
Y_{11} & Y_{12} \\
Y_{21} & Y_{22}
\end{array}\right]
$$

in which $X_{11} \in \mathbb{C}^{r(B) \times r(C)}, X_{12} \in \mathbb{C}^{r(B) \times(q-r(C))}, X_{21} \in$ $\mathbb{C}^{(p-r(B)) \times r(C)}, X_{22} \in \mathbb{C}^{(p-r(B)) \times(q-r(C))}, Y_{11} \in \mathbb{C}^{r(F) \times r(K)}$, $Y_{12} \in \mathbb{C}^{r(F) \times(r(C)-r(K))}, Y_{21} \in \mathbb{C}^{(r(B)-r(F)) \times r(K)}$, and $Y_{22} \in$ $\mathbb{C}^{(r(B)-r(F)) \times(r(C)-r(K))}$. It follows that

$$
\begin{aligned}
& r(A-B X C) \\
& =r\left(U_{1}^{*} A V_{2}-\left[\begin{array}{cc}
\Sigma_{1} & 0 \\
0 & 0
\end{array}\right] V_{1}^{*} X U_{2}\left[\begin{array}{cc}
\Sigma_{2} & 0 \\
0 & 0
\end{array}\right]\right) \\
& =r\left[\begin{array}{cc}
A_{11}-X_{11} & A_{12} \\
A_{21} & A_{22}
\end{array}\right] \\
& =r\left[\begin{array}{cc}
A_{11}-X_{11} & A_{12} V_{3} \\
U_{3}^{*} A_{21} & {\left[\begin{array}{cc}
\Sigma_{3} & 0 \\
0 & 0
\end{array}\right]}
\end{array}\right]
\end{aligned}
$$

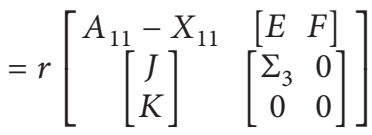

$$
\begin{aligned}
& =r\left[\begin{array}{cc}
A_{11}-E \Sigma_{3}^{-1} J-X_{11} & F \\
K & 0
\end{array}\right]+r\left(A_{22}\right) \\
& =r\left[\begin{array}{cc}
{\left[\begin{array}{cc}
\widehat{A}_{11} & \widehat{A}_{12} \\
\widehat{A}_{21} & \widehat{A}_{22}
\end{array}\right]-U_{4}^{*} X_{11} V_{5}\left[\begin{array}{cc}
\Sigma_{4} & 0 \\
0 & 0
\end{array}\right]} \\
{\left[\begin{array}{cc}
\Sigma_{5} & 0 \\
0 & 0
\end{array}\right]} & 0
\end{array}\right] \\
& +r\left(A_{22}\right)
\end{aligned}
$$




$$
\begin{aligned}
= & r\left[\begin{array}{cccc}
\widehat{A}_{11}-Y_{11} & \widehat{A}_{12}-Y_{12} & \Sigma_{4} & 0 \\
\widehat{A}_{21}-Y_{21} & \widehat{A}_{22}-Y_{22} & 0 & 0 \\
\Sigma_{5} & 0 & 0 & 0 \\
0 & 0 & 0 & 0
\end{array}\right] \\
& +r\left(A_{22}\right) \\
= & r\left(\widehat{A}_{22}-Y_{22}\right)+r\left(A_{22}\right)+r(F)+r(K) .
\end{aligned}
$$

Since $r(A-B X C)=k_{1}$, from (28c), we obtain

$$
r\left(\widehat{A}_{22}-Y_{22}\right)=k_{1}-r\left(A_{22}\right)-r(F)-r(K) .
$$

The identity $r\left(\widehat{A}_{22}-Y_{22}\right)=k_{1}-l$ follows by substituting (24)-(26) into (29). Hence, any solution $Y_{22}$ to the rank equation $r\left(\widehat{A}_{22}-Y_{22}\right)=k_{1}-l$ has the form

$$
Y_{22}=\widehat{A}_{22}+T
$$

where $r(T)=k_{1}-l$.

Substituting (30) into the second partitioned matrix in (27), we obtain

$$
X_{11}=U_{4}\left[\begin{array}{cc}
Y_{11} & Y_{12} \\
Y_{21} & \widehat{A}_{22}+T
\end{array}\right] V_{5}^{*}
$$

where $r(T)=k_{1}-l$. The expression of $X$ in (22) follows by substituting (31) into the first partitioned matrix in (27).

Substituting (24) into the above identity, we have

$$
r\left[\begin{array}{cc}
A_{11}-E \Sigma_{3}^{-1} J & F \\
K & 0
\end{array}\right]=r(A)+r(B)+r(C)-r\left[\begin{array}{ll}
A & B \\
C & 0
\end{array}\right] .
$$

By applying Lemma 3 (9) to the final identity in (23), it follows that

$$
\begin{aligned}
r\left(\widehat{A}_{22}\right) & =r\left(E_{F}\left(A_{11}-E \Sigma_{3}^{-1} J\right) F_{K}\right) \\
& =r\left[\begin{array}{cc}
A_{11}-E \Sigma_{3}^{-1} J & F \\
K & 0
\end{array}\right]-r(F)-r(K) \\
& =r(A)+r\left[\begin{array}{ll}
A & B \\
C & 0
\end{array}\right]-r\left[\begin{array}{l}
A \\
C
\end{array}\right]-r\left[\begin{array}{ll}
A & B
\end{array}\right] \\
& =r(A)-l .
\end{aligned}
$$

We have the following result.

Theorem 8. Let $A, B$, and $C$ be as in Problem 1 and let $k_{1}$ satisfy (18). Then

$$
\min _{r(A-B X C)=k_{1}} r(X)=\max \left\{r(A)-k_{1}, k_{1}-r(A)\right\},
$$

$$
\begin{aligned}
\max _{r(A-B X C)=k_{1}} r(X) \\
=\min \left\{p, q, k_{1}+p+q+r(A)\right. \\
\left.\quad-2 r(B)-2 r(C)+r\left[\begin{array}{l}
A \\
C
\end{array}\right]+r\left[\begin{array}{ll}
A & B
\end{array}\right]\right\} .
\end{aligned}
$$

Proof. From a general expression of $X$ for the rank equation $r(A-B X C)=k_{1}$ given in (22), (10), and (34), we obtain

$$
\begin{aligned}
\min _{r(A-B X C)=k_{1}} r(X) & =\min _{r(T)=k_{1}-l} r\left(\widehat{A}_{22}+T\right) \\
& =\max \left\{k_{1}-l-r\left(\widehat{A}_{22}\right), r\left(\widehat{A}_{22}\right)-k_{1}+l\right\} \\
& =\max \left\{r(A)-k_{1}, k_{1}-r(A)\right\} .
\end{aligned}
$$

From (11), (22), and (34), we obtain

$$
\begin{aligned}
& \max _{r(A-B X C)=k_{1}} r(X)
\end{aligned}
$$

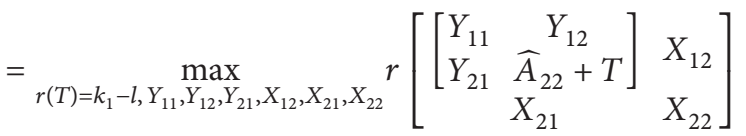

$$
\begin{aligned}
& =\max _{r(T)=k-l, Y \in \mathbb{C}^{p \times q}, Z \in \mathbb{C}^{p \times q}} r\left(\left[\begin{array}{cc}
\widehat{A}_{22}+T & 0 \\
0 & 0
\end{array}\right]-Y\left[\begin{array}{cc}
0 & 0 \\
0 & I_{q-r(C)+r(K)}
\end{array}\right]\right. \\
& \left.-\left[\begin{array}{cc}
0 & 0 \\
0 & I_{p-r(B)+r(F)}
\end{array}\right] Z\right) \\
& =\min \{p, q, p+q \\
& \left.\max _{r(T)=k-l} r\left[\begin{array}{cccc}
\widehat{A}_{22}+T & 0 & 0 & 0 \\
0 & 0 & 0 & I_{p-r(B)+r(F)} \\
0 & 0 & 0 & 0 \\
0 & I_{q-r(C)+r(K)} & 0 & 0
\end{array}\right]\right\}
\end{aligned}
$$




$$
\begin{gathered}
=\min \left\{p, q, \max _{r(T)=k_{1}-l} r\left(\widehat{A}_{22}+T\right)+p+q\right. \\
+r(F)+r(K)-r(B)-r(C)\} \\
=\min \{p, q, p+q+r(K)-r(C), \\
p+q+r(F)-r(B), \\
k_{1}+p+q+r(A)-2 r(B)-2 r(C) \\
\left.+r\left[\begin{array}{l}
A \\
C
\end{array}\right]+r\left[\begin{array}{ll}
A & B
\end{array}\right]\right\} .
\end{gathered}
$$

Since $B \in \mathbb{C}^{m \times p}$ and $C \in \mathbb{C}^{q \times n}$, we see that $p+q+r(K)-r(C) \geq$ $p$ and $p+q+r(F)-r(B) \geq q$. To simplify expression (38) by the two inequalities, we obtain expression (36) for the maximal rank of solutions to the rank equation $r(A-B X C)=k_{1}$.

Remark 9 (see [3]). Let $A, B$, and $C$ be as in Theorem 7. The matrix equation $B X C=A$ is consistent, if and only if there exists $X$ such that $r(A-B X C)=0$. Therefore, applying Theorem 8, we have the extremal ranks of solutions to the matrix equation $B X C=A$ :

$$
\begin{gathered}
\min _{B X C=A} r(X)=r(A), \\
\max _{B X C=A} r(X)=\min \{p, q, p+q+r(A)-r(B)-r(C)\} .
\end{gathered}
$$

Remark 10 (see [9]). Let $A, B$, and $C$ be as in Theorem 7 and let $\mathbb{S}=\left\{X \in \mathbb{C}^{p \times q} \mid\|B X C-A\|=\min \right\}$. Since $X \in \mathbb{S}$, if and only if $B^{*} A C^{*}=B^{*} B X C C^{*}$, and the matrix equation $B^{*} A C^{*}=B^{*} B X C C^{*}$ is always consistent, we can use $B^{*} A C^{*}$, $B^{*} B$, and $C C^{*}$ to replace $A, B$, and $C$ in (39). Then we have the extremal ranks of least squares solutions of the matrix equation $B X C=A$ :

$$
\begin{gathered}
\min _{\|B X C-A\|=\min } r(X)=r\left(B^{*} A C^{*}\right), \\
\max _{\|B X C-A\|=\min } r(X)=\min \{p, q, p+q \\
\left.+r\left(B^{*} A C^{*}\right)-r(B)-r(C)\right\} .
\end{gathered}
$$

In [14], Liu and Tian derive the extremal ranks of submatrices in a Hermitian solution to the consistent matrix equation $B X B^{*}=A$. In the following theorem, we derive the range of $k_{1}$ such that there exists a Hermitian solution $X$ to the rank equation $r\left(A-B X B^{*}\right)=k_{1}$, and the maximal and minimal ranks of $X$ which may be proved in the same way as Theorem 8 .
Theorem 11. Let $A \in \mathbb{C}^{m \times m}$ and $B \in \mathbb{C}^{m \times n}$ be given, and let $A$ be Hermitian. Then from [15] there exists a Hermitian matrix $X$ satisfying $r\left(A-B X B^{*}\right)=k_{1}$, if and only if

$$
r\left[\begin{array}{ll}
A & B
\end{array}\right] \geq k_{1} \geq 2 r\left[\begin{array}{ll}
A & B
\end{array}\right]-r\left[\begin{array}{cc}
A & B \\
B^{*} & 0
\end{array}\right] .
$$

If $k_{1}$ satisfies the above inequalities, then

$$
\begin{array}{r}
\min _{r\left(A-B X B^{*}\right)=k_{1}} r(X)=\max \left\{r(A)-k_{1}, k_{1}-r(A)\right\}, \\
\max _{r\left(A-B X B^{*}\right)=k_{1}} r(X)=\min \left\{n, k_{1}+2 n+r(A)\right. \\
\left.-4 r(B)+2 r\left[\begin{array}{ll}
A & B
\end{array}\right]\right\} .
\end{array}
$$

\section{Solutions to Problem 2}

In this section, we study Problem 2 proposed in Section 1.

Let

$$
A=\left[\begin{array}{ll}
1 & 0 \\
0 & 0
\end{array}\right], \quad B=C=I_{2} .
$$

It is obvious that

$$
2 \geq r(A-B X C) \geq 0
$$

and there do not exist the least squares solutions of $\|B X C-A\|_{F}=$ min subject to $r(A-B X C)=2$. Therefore, we should study the range of $k$, such that there exists a least squares solution $X$ of $\|B X C-A\|_{F}=$ min subject to $r(A-$ $B X C)=k$.

Theorem 12. Let $A, B$, and $C$ be as in Theorem 7. Then there exists a least squares solution of $\|A-B X C\|=\min$ under the rank constraint $r(A-B X C)=k$, if and only if

$$
\begin{gathered}
r\left[\begin{array}{ccc}
A & A C^{*} & B \\
B^{*} A & 0 & 0 \\
C & 0 & 0
\end{array}\right]-r(B)-r(C) \geq k \\
\geq r\left[\begin{array}{l}
A \\
C
\end{array}\right]+r\left[\begin{array}{ll}
A & B
\end{array}\right]-r\left[\begin{array}{ll}
A & B \\
C & 0
\end{array}\right] .
\end{gathered}
$$

Proof. Let $E, F, J, K, \Sigma_{i}, U_{i}$, and $V_{i}(i=1,2,3,4,5)$ be as in Theorem 7, and let $U_{4}^{*}\left(E \Sigma_{3}^{-1} J\right) V_{5}$ be partitioned in the form

$$
U_{4}^{*}\left(E \Sigma_{3}^{-1} J\right) V_{5}=\left[\begin{array}{ll}
D_{11} & D_{12} \\
D_{21} & D_{22}
\end{array}\right],
$$

where $D_{22} \in \mathbb{C}^{(r(B)-r(F)) \times(r(C)-r(K))}, D_{21} \in \mathbb{C}^{(r(B)-r(F)) \times r(K)}$, $D_{12} \in \mathbb{C}^{r(F) \times(r(C)-r(K))}$, and $D_{11} \in \mathbb{C}^{r(F) \times r(K)}$. Let $D_{22}$ have the singular value decomposition

$$
D_{22}=U_{7}\left[\begin{array}{cc}
\Sigma_{7} & 0 \\
0 & 0
\end{array}\right] V_{7}^{*}
$$

where $\Sigma_{7}=\operatorname{diag}\left\{\delta_{1}, \ldots, \delta_{r\left(D_{22}\right)}\right\}, \delta_{1} \geq \cdots \geq \delta_{r\left(D_{22}\right)}>0$, and $U_{7}$ and $V_{7}$ are unitary matrices of appropriate sizes. 
From the partitioned form for $U_{4}^{*}\left(A_{11}-E \Sigma_{3}^{-1} J\right) V_{5}$ in (21),

$$
U_{4}^{*} A_{11} V_{5}=U_{4}^{*}\left(E \Sigma_{3}^{-1} J\right) V_{5}+\left[\begin{array}{ll}
\widehat{A}_{11} & \widehat{A}_{12} \\
\widehat{A}_{21} & \widehat{A}_{22}
\end{array}\right] .
$$

Since the Frobenius norm is invariant, we have the following identities by substituting (22) into $\|A-B X C\|$ and applying (46) and (48):

$$
\begin{aligned}
& \min _{r(A-B X C)=k}\|A-B X C\|^{2} \\
= & \min _{Y_{11}, Y_{12}, Y_{21}, r(T)=k-l} \|\left[\begin{array}{cc}
A_{11}-U_{4}\left[\begin{array}{cc}
Y_{11} & Y_{12} \\
Y_{21} & \widehat{A}_{22}+T \\
A_{21}
\end{array}\right] V_{5}^{*} & A_{12} \\
= & \left\|\left[\begin{array}{cc}
0 & A_{12} \\
A_{21} & A_{22}
\end{array}\right]\right\|^{2}
\end{array}\right. \\
& +\underset{Y_{11}, Y_{12}, Y_{21}, r(T)=k-l}{ }\left\|U_{4}^{*} A_{11} V_{5}-\left[\begin{array}{cc}
Y_{11} & Y_{12} \\
Y_{21} & \widehat{A}_{22}+T
\end{array}\right]\right\|^{2} \\
= & \left\|\left[\begin{array}{cc}
0 & A_{12} \\
A_{21} & A_{22}
\end{array}\right]\right\|^{2} \\
& +\underset{Y_{11}, Y_{12}, Y_{21}, r(T)=k-l}{\min _{2}}\left\|U_{4}^{*}\left(E \Sigma_{3}^{-1} J\right) V_{5}-\left[\begin{array}{cc}
Y_{11} & Y_{12} \\
Y_{21} & T
\end{array}\right]\right\|^{2} \\
= & \left\|\left[\begin{array}{cc}
0 & A_{12} \\
A_{21} & A_{22}
\end{array}\right]\right\|^{2}+\underset{r(T)=k-l}{\min }\left\|D_{22}-T\right\|^{2} .
\end{aligned}
$$

Therefore, there exists a least squares solution $X$ satisfying $\|A-B X C\|^{2}=$ min subject to $r(A-B X C)=k$ if and only if $r\left(D_{22}\right) \geq r(T) \geq 0$, that is, if and only if

$$
r\left(D_{22}\right)+l \geq k \geq l \text {. }
$$

From the partitioned form for $U_{4}^{*}\left(E \Sigma_{3}^{-1} J\right) V_{5}$ in (46) and the decompositions of $F$ and $K$ in (21), we have $r\left(D_{22}\right)=$ $r\left(E_{F} E \Sigma_{3}^{-1} J F_{K}\right)$. Applying (9) gives

$$
r\left(D_{22}\right)=r\left[\begin{array}{cc}
E \Sigma_{3}^{-1} J & F \\
K & 0
\end{array}\right]-r(F)-r(K) .
$$

The identity

$$
r\left(A-B B^{\dagger} A C^{\dagger} C\right)=r\left[\begin{array}{cc}
0 & A_{12} \\
A_{21} & A_{22}
\end{array}\right]
$$

follows from applying the decompositions of $B$ and $C$ in (19) and the partitioned form for $U_{1}^{*} A V_{2}$ in (20). Substituting the decomposition of $A_{22}$ in (19) into $\left[\begin{array}{cc}0 & A_{12} \\ A_{21} & A_{22}\end{array}\right]$, applying the partitioned forms for $U_{3}^{*} A_{21}$ and $A_{12} V_{3}$ in (21), we conclude that

$$
r\left(A-B B^{\dagger} A C^{\dagger} C\right)=r\left[\begin{array}{cc}
E \Sigma_{3}^{-1} J & F \\
K & 0
\end{array}\right]+r\left(A_{22}\right) .
$$

Hence,

$$
r\left(D_{22}\right)=r\left(A-B B^{\dagger} A C^{\dagger} C\right)-r\left(A_{22}\right)-r(F)-r(K) .
$$

It follows from applying (12) that

$$
r\left(A-B B^{\dagger} A C^{\dagger} C\right)=r\left[\begin{array}{ccc}
A & A C^{*} & B \\
B^{*} A & 0 & 0 \\
C & 0 & 0
\end{array}\right]-r(B)-r(C) .
$$

Substituting (24)-(26) and (55) into (54), we have

$$
\begin{aligned}
r\left(D_{22}\right)= & r\left[\begin{array}{ccc}
A & A C^{*} & B \\
B^{*} A & 0 & 0 \\
C & 0 & 0
\end{array}\right]+r\left[\begin{array}{ll}
A & B \\
C & 0
\end{array}\right] \\
& -r\left[\begin{array}{l}
A \\
C
\end{array}\right]-r\left[\begin{array}{ll}
A & B
\end{array}\right]-r(B)-r(C) .
\end{aligned}
$$

Therefor, the inequalities in (45) follow from substituting (17) and (56) into (50).

Theorem 13. Let $A, B, C, E, F, J, K, \Sigma_{i}, U_{i}$, and $V_{i}(i=$ $1,2,3,4,5)$ be as in Theorem 7 , and let $U_{4}^{*}\left(E \Sigma_{3}^{-1} J\right) V_{5}$ and $D_{22}$ be partitioned as in (46) and (47), respectively. If $k$ satisfies (45), then any least squares solution $X \in\{X \mid r(A-B X C)=k\}$ satisfying $\|A-B X C\|=\min$ has the form

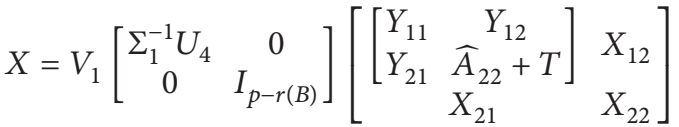

$$
\begin{aligned}
& \times\left[\begin{array}{cc}
V_{5}^{*} \Sigma_{2}^{-1} & 0 \\
0 & I_{q-r(C)}
\end{array}\right] U_{2}^{*} \text {, }
\end{aligned}
$$

where $X_{12} \in \mathbb{C}^{r(B) \times(q-r(C))}, X_{21} \in \mathbb{C}^{(p-r(B)) \times r(C)}, X_{22} \in$ $\mathbb{C}^{(p-r(B)) \times(q-r(C))}, Y_{11} \in \mathbb{C}^{r(F) \times r(K)}, Y_{12} \in \mathbb{C}^{r(F) \times(r(C)-r(K))}, Y_{21} \in$ $\mathbb{C}^{(r(B)-r(F)) \times r(K)}$, and $T \in \mathbb{C}^{(r(B)-r(F)) \times(r(C)-r(K))}$ are arbitrary matrices, such that $r(T)=k-l$.

(1) When $\delta_{k-l}>\delta_{k-l+1}$,

$$
T=U_{7} \operatorname{diag}\left\{\delta_{1}, \ldots, \delta_{k-l}, 0, \ldots, 0\right\} V_{7}^{*} ;
$$

(2) when $\widehat{p}<k-l<\widehat{q} \leq r$ and $\lambda_{\widehat{p}}>\lambda_{\widehat{p}+1}=\cdots=\delta_{\widehat{q}}>$ $\delta_{\widehat{q}+1}$,

$$
T=U_{7} \operatorname{diag}\left\{\delta_{1}, \ldots, \delta_{\hat{p}}, \delta_{k-l} Q Q^{*}, 0, \ldots, 0\right\} V_{7}^{*},
$$

where $Q$ is an arbitrary matrix satisfying $Q \in \mathbb{C}^{(\hat{q}-\hat{p}) \times(k-l-\hat{p})}$ and $Q^{*} Q=I_{k-l-\widehat{p}}$.

Proof. When $k$ satisfies the inequalities in (45), then, by applying Lemma 6 and (48), we obtain the desired form of $T$ in (58) and (59), respectively.

\section{Conclusions}

In this paper, we have discussed the solutions to Problem 1

$$
\mathbb{S}_{k_{1}}=\left\{X \mid X \in \mathbb{C}^{p \times q}, r(A-B X C)=k_{1}\right\}
$$


and the solutions to Problem 2

$$
\widehat{\mathbb{S}}=\left\{X \mid X \in \mathbb{S}_{k},\|A-B X C\|^{2}=\min \right\} .
$$

We first derived the expression of solutions to $r(A-$ $B X C)=k_{1}$ when Problem 1 is solvable. Based on these results, we obtained the extremal ranks of the expression of solutions to Problem 1, the solvability conditions of Problem 2, and the expression of least squares solutions when Problem 2 is solvable.

\section{Conflict of Interests}

The authors declare that there is no conflict of interests regarding the publication of this paper.

\section{Acknowledgments}

The authors would like to thank the referees for their helpful comments and suggestions. The work of the first author was supported in part by the National Natural Science Foundation of China (Grant no. 11171226). The work of the second author was supported in part by the University Natural Science Foundation of Anhui Province (Grant no. KJ2013A239) and the National Natural Science Foundation of China (Grant no. 11301529).

\section{References}

[1] A. Ben-Israel and T. N. E. Greville, Generalized Inverses: Theory and Applications, Springer, Berlin, Germany, 2nd edition, 2003.

[2] F. Uhlig, "On the matrix equation $A X=B$ with applications to the generators of a controllability matrix," Linear Algebra and its Applications, vol. 85, pp. 203-209, 1987.

[3] Y. Tian, "Ranks of solutions of the matrix equation $A X B=C$," Linear and Multilinear Algebra, vol. 51, no. 2, pp. 111-125, 2003.

[4] R. Li and Y. Liu, "Ranks of Hermitian solutions of the matrix equation $A X=B$," Far East Journal of Mathematical Sciences, vol. 26, no. 1, pp. 117-126, 2007.

[5] Y. Li, F. Zhang, W. Guo, and J. Zhao, "Solutions with special structure to the linear matrix equation $A X=B$," Computers \& Mathematics with Applications, vol. 61, no. 2, pp. 374-383, 2011.

[6] Y. H. Liu, "Ranks of solutions of the linear matrix equation $A X+$ $Y B=C$," Computers \& Mathematics with Applications, vol. 52, no. 6-7, pp. 861-872, 2006.

[7] Q.-W. Wang and C.-K. Li, "Ranks and the least-norm of the general solution to a system of quaternion matrix equations," Linear Algebra and Its Applications, vol. 430, no. 5-6, pp. 1626$1640,2009$.

[8] Q. Wang and Z. H. He, "Solvability conditions and general solution for mixed Sylvester equations," Automatica, vol. 49, no. 9, pp. 2713-2719, 2013.

[9] Y. H. Liu, "Ranks of least squares solutions of the matrix equation $A X B=C$," Computers \& Mathematics with Applications, vol. 55, no. 6, pp. 1270-1278, 2008.

[10] K. C. Sou and A. Rantzer, "On a generalized matrix approximation problem in the spectral norm," Linear Algebra and Its Applications, vol. 436, no. 7, pp. 2331-2341, 2012.

[11] M. Wei and D. Shen, "Minimum rank solutions to the matrix approximation problems in the spectral norm," SIAM Journal on Matrix Analysis and Applications, vol. 33, no. 3, pp. 940-957, 2012.

[12] X. F. Duan, Q. W. Wang, and J. F. Li, "On the low-rank approximation arising in the generalized Karhunen-Loeve transform," Abstract and Applied Analysis, vol. 2013, Article ID 528281, 8 pages, 2013.

[13] H. Wang and J. Xu, "Some results on characterizations of matrix partial orderings," Journal of Applied Mathematics, vol. 2014, Article ID 408457, 6 pages, 2014.

[14] Y. Liu and Y. Tian, "Extremal ranks of submatrices in an Hermitian solution to the matrix equation $A X A^{*}=B$ with applications," Journal of Applied Mathematics and Computing, vol. 32, no. 2, pp. 289-301, 2010.

[15] Y. Tian and Y. Liu, "Extremal ranks of some symmetric matrix expressions with applications," SIAM Journal on Matrix Analysis and Applications, vol. 28, no. 3, pp. 890-905, 2006.

[16] H. Wang, "On least squares solutions subject to a rank restriction," Linear and Multilinear Algebra, 2014.

[17] M. Wei, "Perturbation theory for the Eckart-Young-Mirsky theorem and the constrained total least squares problem," Linear Algebra and Its Applications, vol. 280, no. 1-3, pp. 267287,1998

[18] H. Y. Zha, "The restricted singular value decomposition of matrix triplets," SIAM Journal on Matrix Analysis and Applications, vol. 12, no. 1, pp. 172-194, 1991.

[19] M. Wei and Q. Wang, "On rank-constrained Hermitian nonnegative-definite least squares solutions to the matrix equation $A X A H=B$," International Journal of Computer Mathematics, vol. 84, no. 6, pp. 945-952, 2007.

[20] G. Marsaglia and G. P. H. Styan, "Equalities and inequalities for ranks of matrices," Linear and Multilinear Algebra, vol. 2, pp. 269-292, 1974.

[21] Y. Tian, "More on maximal and minimal ranks of Schur complements with applications," Applied Mathematics and Computation, vol. 152, no. 3, pp. 675-692, 2004.

[22] C. Eckart and G. Young, "The approximation of one matrix by another of lower rank," Psychometrika, vol. 1, no. 3, pp. 211-218, 1936.

[23] L. Mirsky, "Symmetric gauge functions and unitarily invariant norms," The Quarterly Journal of Mathematics, vol. 11, pp. 50-59, 1960.

[24] G. H. Golub and C. F. van Loan, Matrix computations, vol. 3 of Johns Hopkins Series in the Mathematical Sciences, Johns Hopkins University Press, Baltimore, Md, USA, 2nd edition, 1989.

[25] D. L. Chu, H. C. Chan, and D. W. C. Ho, "Regularization of singular systems by derivative and proportional output feedback," SIAM Journal on Matrix Analysis and Applications, vol. 19, no. 1, pp. 21-38, 1998. 


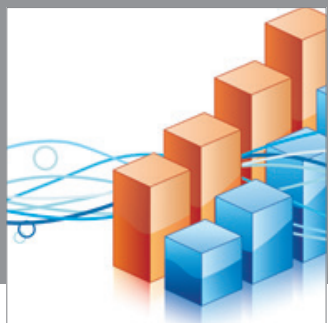

Advances in

Operations Research

mansans

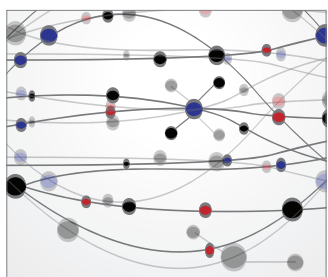

The Scientific World Journal
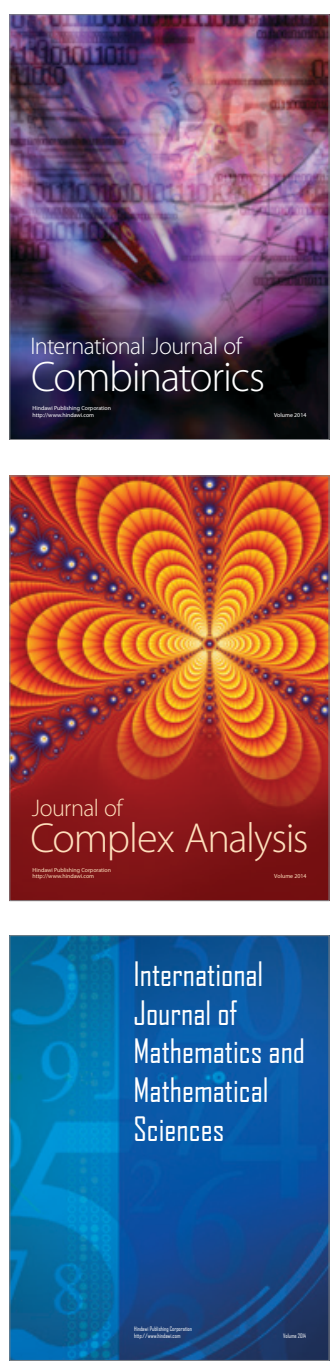
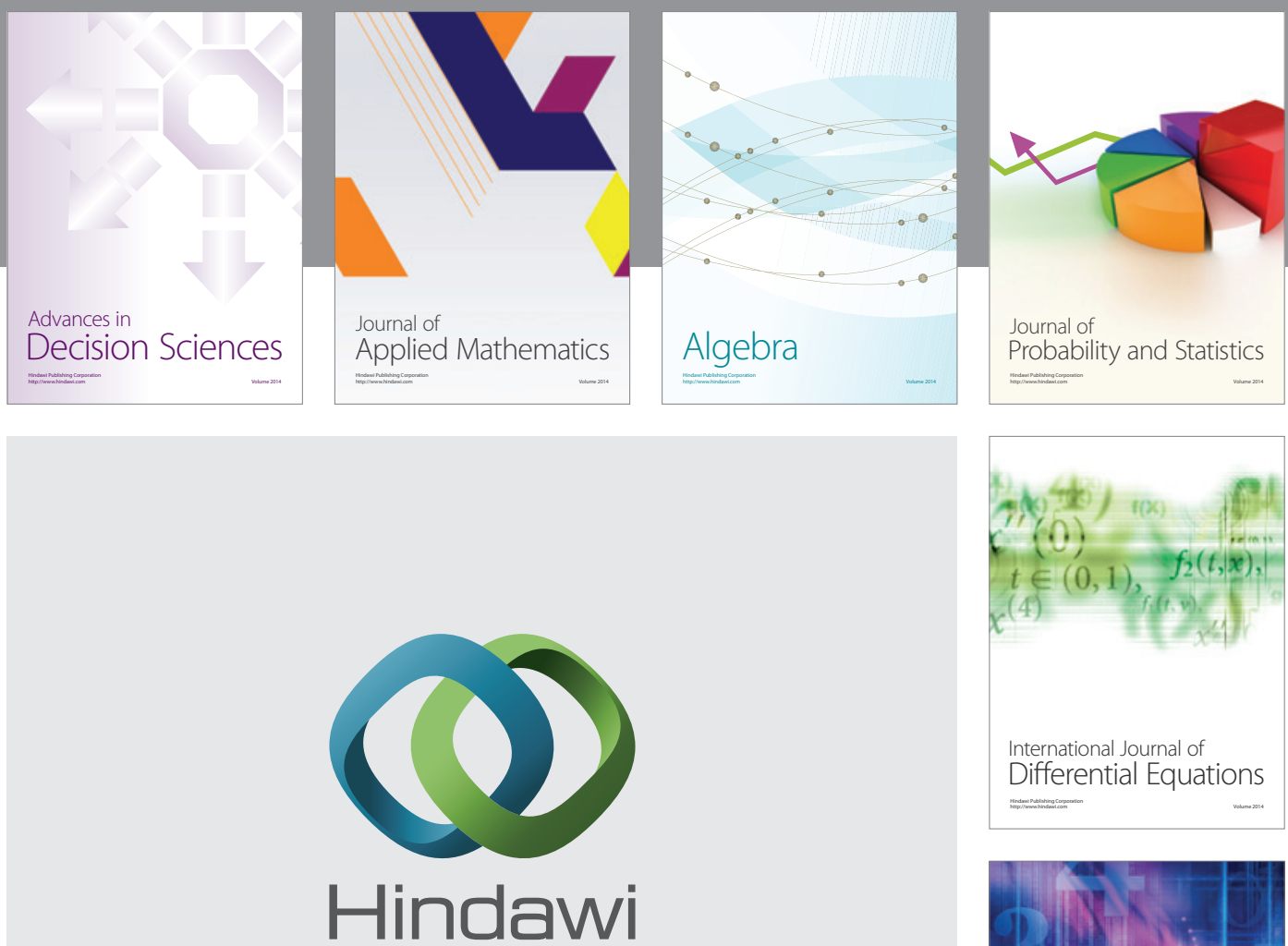

Submit your manuscripts at http://www.hindawi.com
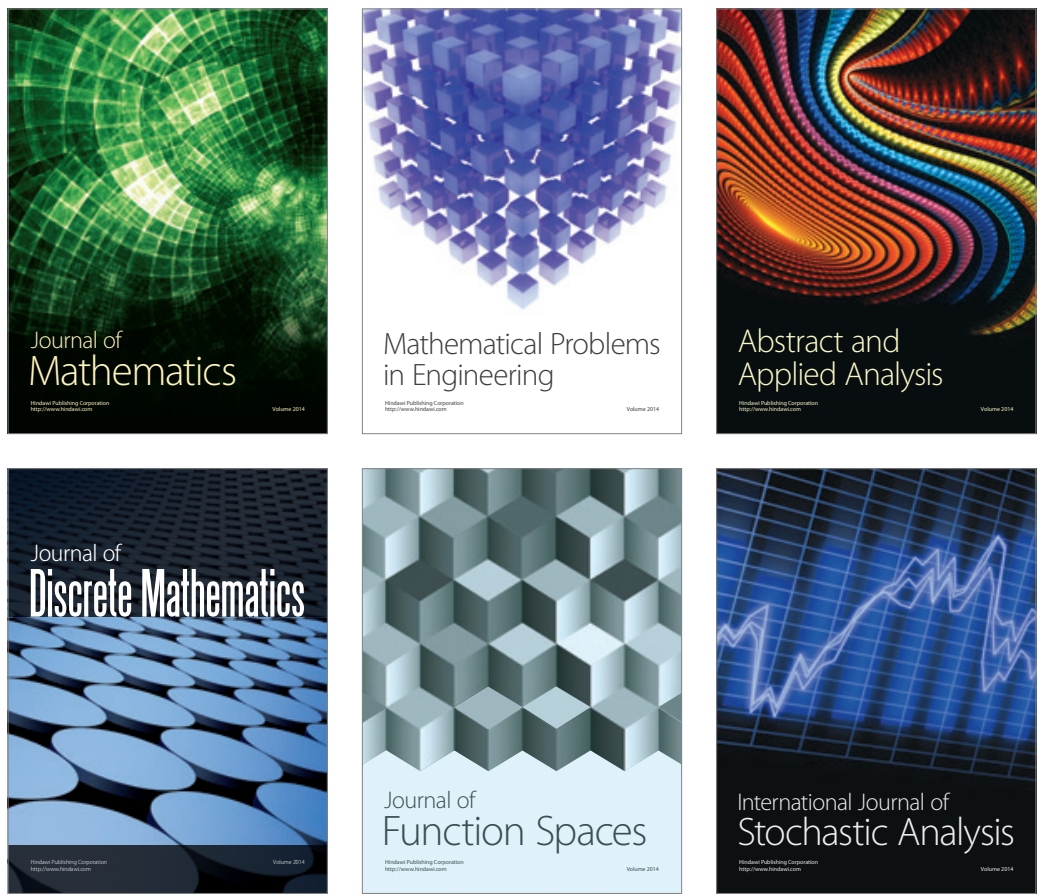

Journal of

Function Spaces

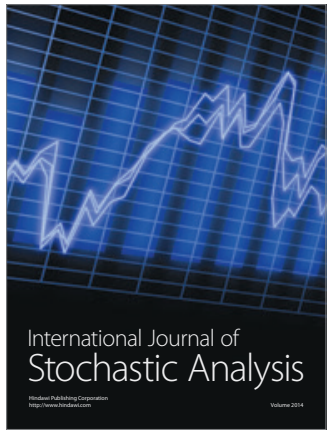

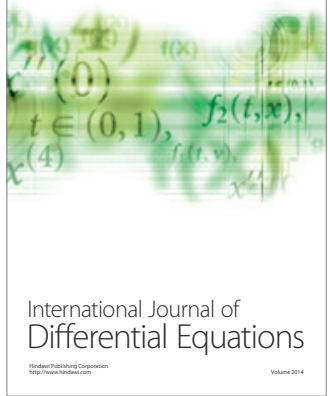
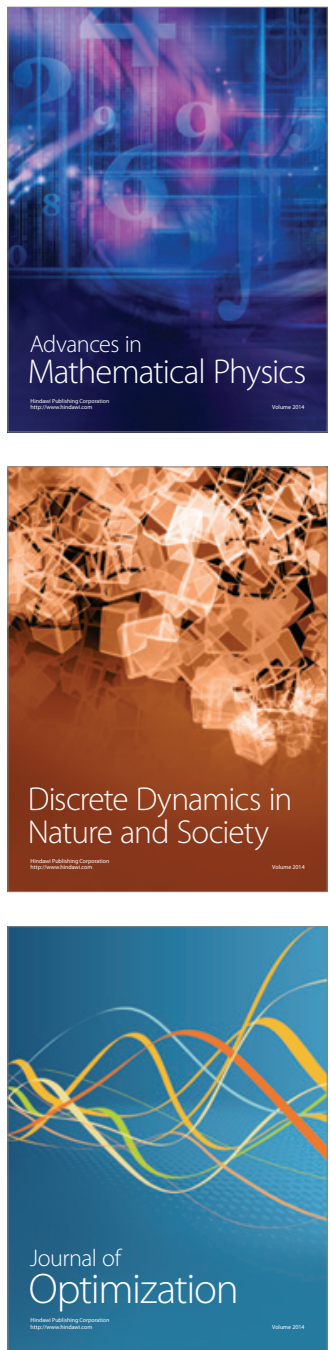